\title{
Historique de la loi sur l'eau du 3 janvier 1992 : les SDAGE et les SAGE
}

\author{
Jean-Loïc Nicolazo \\ Chargé de mission auprès du Directeur de l'Eau, Ministère de l'environnement
}

L'idée d'organiser la gestion de l'eau à partir d'une vision prospective n'est pas nouvelle. Plusieurs éléments concourent à une telle démarche. Ils résultent d'une problématique incontournable propre à cet élément particulier que constitue l'eau et qui tiennent à :

- la variété des intérêts, souvent porteurs de conflits, qui s'attachent à l'usage de l'eau,

- la multiplicité des décideurs, publics ou privés, qui participent de manière directe ou indirecte à la co-gestion de l'eau,

- le poids des investissements nécessaires à la satisfaction des usages collectifs qui peuvent rarement s'insérer dans les limites d'une annualité budgétaire.

Ce « désordre » est peu compatible avec une gestion des eaux qui se veut à la fois cohérente et rationnelle. D'où la recherche de mécanismes ou procédures de planification, de programmation ou de contractualisation qui se veulent organiser des règles du jeu avec des partenaires associés dans un projet commun où chacun a son rôle à jouer.

\section{LES PREMICES}

Ce sont les schémas d'Aménagement des Eaux institués par la circulaire du 19 juillet 1978.

La circulaire du ministre de l'environnement et du cadre de vie du 19 juillet 1978 s'adressait aux préfets de régions et aux préfets coordonnateurs de bassin, présidents des missions déléguées de bassin. Le dispositif mis en place était le suivant :

- au niveau national, le Comité interministériel d'action pour la nature et l'environnement (C.I.A.N.E.) avait approuvé le 14 février 1978 les lignes directrices d'un schéma à long terme de développement des ressources en eau et de la reconquête de leur qualité,

- au niveau local, qui pouvait être le bassin, le sousbassin ou toute entité régionale adéquate, il était suggéré d'établir en tant que de besoin des schémas d'aménagement des eaux.

Ces schémas avaient pour fonction principale de fixer les priorités à moyen terme dans les domaines de la satisfaction des besoins et des niveaux de qualité à atteindre. Leur rôle était de servir de guide dans la programmation des investissements et des décisions de police des eaux. Des alternatives étaient possibles et des priorités devaient être dégagées, assorties d'une évaluation financière.

L'élaboration de ces documents était confiée aux services de l'Etat, sous la responsabilité des missions déléguées de bassin, qui devaient veiller à la cohérence des Schémas d'Aménagement des Eaux au niveau de chaque bassin. Les Comités de Bassin étaient appelés à émettre un avis.

Ces schémas d'aménagement des eaux n'ont pas rencontré le succès escompté.

Plusieurs raisons à cet échec :

- leur caractère juridiquement imprécis, sans valeur juridique définie,

- l'implication réduite des élus locaux dans une démarche à géométrie variable, peu motivante pour eux.

- un processus et une procédure trop exclusivement administratifs et dont le fondement restait réduit à une circulaire.

\section{IES SDAGE ET LES SAGE}

La théorie a été initiée par la loi sur l'eau du 3 janvier 1992. 
Le législateur de 1992 reprit l'idée mais dans une approche tout à fait innovante.

Au préalable, il paraît utile de rappeler que le texte du projet de loi déposé par le gouvernement introduisait, dans une nouvelle forme, seulement la notion de Schémas d'Aménagement et de Gestion des Eaux. C'est au cours de débats à l'Assemblée Nationale que la notion de Schémas Directeurs d'Aménagement et de Gestion des Eaux est apparue. L'introduction de cet élément nouveau dans l'architecture prévue à l'origine, a obligé à revoir assez fondamentalement l'approche des SAGE. Une question essentielle devait rapidement être résolue : celle de la place respective des SAGE par rapport aux SDAGE.

En introduisant le mécanisme des SDAGE, l'idée était de veiller à organiser les SAGE dans un cadre de référence garantissant une cohérence à la politique de l'eau dans le bassin. Les SAGE, d'initiative locale, établis à l'échelle d'une vallée, d'une rivière ou d'un sous-bassin, se voient encadrés par des SDAGE au niveau du bassin hydrographique. On aboutit ainsi à un système à deux niveaux rappelant, à leur échelle, le système de planification de l'espace propre à l'urbanisme: les Schémas Directeurs encadrant les Plans d'Occupation des Sols. Une différence notable toutefois, significative, à bien des égards : la loi de 1992 rend obligatoire l'élaboration des SDAGE et ce, dans un délai de 5 ans. Tout laisse à penser que cette échéance sera largement respectée.

Autre différence, fondamentale elle aussi, est que SDAGE et SAGE ne sont pas des documents qui aboutissent, comme c'est le cas en matière d'urbanisme, à reconnaître des compétences nouvelles aux élus locaux. Ils ne s'inscrivent pas dans une démarche de décentralisation et n'organisent pas une nouvelle répartition des pouvoirs entre l'Etat et les collectivités locales. La co-gestion existait en pratique depuis longtemps et il y a eu consensus pour ne pas en modifier les termes

En dehors de leur niveau d'intervention différent, les SDAGE et les SAGE ne bénéficient pas du même degré de précision de la part du législateur. La démarche des SAGE est très détaillée par l'article 5 de la loi qui renvoie, en tant que de besoin à un décret (il s'agit du décret n ${ }^{\circ}$ 92-1042 du 24 septembre 1992). La procédure de création de la commission locale de l'eau chargée d'élaborer le $S A G E$, la fixation de son périmètre, le recours éventuel à une communauté locale de l'Eau (décret 4094-289 du 6 avril 1994) sont organisés par un ensemble complet de dispositions. Le dispositif organisé pour les SDAGE est beaucoup plus succint. L'article 3 de la loi qui en constitue le fondement ne renvoie pas à un décret. C'est par voie de circulaires ou de notes d'information qu'une doctrine administrative s'est forgée pour répondre aux questions ou aux difficultés rencontrées dans l'établissement des SDAGE, au regard en particulier de leur représentation locale que devront être les SAGE.

\section{LES SDAGE}

Ils apportent les réponses aux questions posées.

Une première série de questions relève de la place à affecter aux SDAGE par rapport aux autres outils de programmation ou de planification dans le domaine de l'eau.

Première réponse évidente: un SDAGE n'est pas un SAGE.
Si le législateur a décidé d'une double approche géographique, c'est qu'il a souhaité qu'à l'un et l'autre des niveaux soit réservée une analyse adaptée. En conséquence :

- Le SDAGE peut déterminer des périmètres de SAGE et peut inciter à leur émergence, là où des priorités s'imposent.

- Le SDAGE ne peut se substituer à un SAGE dans tous les compartiments de la gestion de l'eau à l'échelle d'un sous-bassin ou d'une rivière.

- il faut donc que le SDAGE observe une certaine « distanciation » pour ne pas, à son échelle, «étouffer » toute chance à des SAGE de se manifester.

Une autre série de questions concerne la valeur normative du SDAGE en tant qu'outil de cohérence ou de contrainte vis-à-vis de la police de l'eau et des milieux aquatiques.

Il y a lieu sans doute de rappeler que la police des eaux (comme toute police administrative) relève de l'autorité directe de l'Etat. Elle permet, à titre exceptionnel, à l'Administration de réduire, limiter ou supprimer des droits qui sont normalement garantis par la loi, dans un but d'intérêt général. De ce fait, les prescriptions que l'Administration peut édicter sont encadrées par des dispositions législatives, des décrets ou des arrêtés. Ces prescriptions sont édictées au niveau central ou au niveau départemental (préfets), dans le respect de procédures organisées réglementairement.

- Le SDAGE ne peut donc, à son niveau, réduire des droits existants, modifier des procédures ou empiéter sur des niveaux de déconcentration organisés par ailleurs. II doit en particulier exclure toutes normes individuelles. Toutefois, sous le contrôle éventuel du juge administratif, les décisions et autorisations prises au titre de la police des eaux devront être compatibles avec les orientations du SDAGE.

- Le SDAGE peut en revanche dégager des principes, des orientations, des priorités, des positions communes dans les champs d'appréciation ouverts par les règlements aux représentants de l'Etat (dans la majeure partie des cas, les préfets) et qui vont pouvoir encadrer l'exercice de la police de l'eau (ou d'autres polices administratives interférentes : schémas de carrière, schémas de déchets, etc...). - Le SDAGE peut, de ce point de vue, s'inscrire dans une perspective de temps déterminée et afficher un objectif de politique générale, dès lors que l'Etat en phase ultime d'approbation adhèrera, par sa signature, à telle ou telle orientation qui lui aura été proposée. Ce serait par exemple le cas d'une politique de révision, d'adaptation ou d'annulation de concessions hydrauliques, à condition qu'elle s'inscrive dans le respect des dispositions législatives et réglementaires prévues. Ce type d'orientation n'est pas à exclure mais pour être crédible doit englober l'ensemble du dispositif prévu, y compris son coût...

Troisième, enfin, série de questions, celle du réalisme économique.

Il faut d'emblée considérer que les SDAGE doivent s'inscrire dans un espace-temps déterminé. En même temps, le SDAGE n'est pas un outil de planification au sens traditionnel du terme (c'est-à-dire nécessitant une adoption par voie législative) ; il ne peut pourtant, pour des raisons de crédibilité des «orientations fondamentales » qu'il a à arrêter, éviter de comporter un volet économique. Il ne s'agit pas d'établir une évaluation du coût global du 
SDAGE dans tous ses compartiments. Par contre, les domaines qui peuvent faire l'objet d'une évaluation économique doivent faire l'objet d'une présentation adéquate.

Le SDAGE, parallèlement, doit s'inscrire soit globalement, soit vis-à-vis de telle priorité, dans une perspective de temps : a priori les dix ou quinze prochaines années. Les évaluations économiques peuvent rarement être crédibles au-delà. Parallèlement, il est indispensable, qu'en ce qui les concerne, les Agences cadrent les perspectives de leur $\mathrm{VII}^{\mathrm{e}}$ programme d'intervention en cohérence avec le futur SDAGE.

Il est logique que, vis-à-vis au moins de ces deux seuls outils de référence existant au niveau de chaque bassin, élaborés par les mêmes responsables, la recherche d'une compatibilité forte soit revendiquée par l'ensemble des partenaires.

En définitive, qu'est-ce que doit être un SDAGE?

Un SDAGE peut se définir comme un document d'orientation (règle du jeu, contrat d'objectif, charte...) par lequel, après une analyse en commun, l'ensemble des partenaires conviennent d'objectifs communs à l'échelle du bassin, à échéance de 10 à 15 ans au plus.

Chaque partenaire - Etat, collectivité territoriale, représentants des usagers —, ne peut jouer le rôle qui lui appartient que dans la limite des pouvoirs et compétences qui lui sont reconnus par les lois et règlements.

Chacun des partenaires est en outre amené à afficher, vis-à-vis des autres mais aussi des tiers, ses options visant «sa» politique de l'eau pour les années à venir, et de la rendre cohérente (compatible) avec celle des autres. Par exemple il incite l'Etat à afficher sa politique de police de l'eau pour les années à venir et chaque usager est en mesure de connaître globalement, les contraintes qui sont susceptibles de lui être imposées.

Ainsi le SDAGE a vocation à l'échelle dı bassin, de constituer le document de référence devant être repris par :

- les politiques sectorielles de l'eau animées par tel ou tel partenaire (eau potable, assainissement, pêche...).

- les politiques de l'eau animées à l'échelle inférieure soit au niveau des collectivités territoriales, soit au niveau d'un sous-bassin avec les SAGE, les contrats de rivière, etc...

A ce titre le SDAGE fixe au niveau du bassin les orientations fondamentales d'une politique de l'eau que chacun aura à respecter, à son niveau, au regard des compétences qui lui sont reconnues et des décisions qu'il sera amené à prendre. 